\title{
A Multi-LeVel COMPUTING ARCHITECTURE FOR EMBEDDED MULTIMEDIA APPLICATIONS
}

We describe and evaluate a template architecture for SoC systems intended for multimedia applications. The architecture is a 2-level hierarchy that consists at the bottom level of several processing units (PUs), controlled at the top level by a control processor. The main characteristic of our architecture is that it exploits in hardware parallelism among tasks executing on different PUs in the same way a superscalar processor exploits instruction level parallelism. This hardware support for task-level parallelism gives rise to a natural programming model that relieves programmers from explicitly synchronizing tasks and communicating data. We describe a number of code transformations to port and improve performance; these transformations are based on well-known compiler analyses, and thus can be incorporated into a compiler for this architecture. We use simulation, two realistic multimedia applications and the proposed code transformations to explore the performance vs. resources trade-off.
Faraydon Karim(1), Alain Mellan(1), Anh Nguyen(1), Utku Aydonat(2), Tarek Abdelrahman(2)

(1) STMicroelectronics

(2) Edward S. Rogers Sr. Department of Electrical and Computer Engineering University of Toronto
Keywords: task-level parallelism, superscalar processors, multiprocessors, compiler optimizations, heterogeneous processors

\section{INTRODUCTION}

A solution paradigm that has emerged in the embedded systems market over the last few years is that of a programmable System-on-a-Chip (SoC), an integrated design that incorporates programmable cores, custom or semi-custom blocks, and memories into a single chip. This paradigm allows the reuse of pre-designed cores (commonly referred to as intellectual property, or IP), thus amortizing the design cost of a core over many system generations.

In this context, we designed a novel architecture to simplify integration of heterogeneous IP for multimedia and streaming applications. The Multi-Level Computing Architecture (MLCA) is a template architecture that features multiple processing units and a top level controller that automatically exploits parallelism among coarse-grain units of computation, or tasks, using well-developed superscalar principles. The MLCA supports a programming model which, similar to that of sequential programming, does not require programmers to specify synchronization and/or data

This work was supported by a research grant from STMicroelectronics. 
communication. Compiler technology to port and improve the performance of MLCA programs is developed at the same time as the architectural design is completed.

In this paper, we describe the MLCA and its programming model. In particular, we explore the benefits of the architecture and use two realistic multimedia applications to show that minimal effort is needed in porting these applications to an instance of MLCA. We further describe a set of code transformations for porting and improving performance of programs on the MLCA. These transformations are based on standard compiler analyses and hence, can be incorporated into compilers for the MLCA. We use simulation, the two applications, and the proposed transformations to explore the performance vs. resources trade-off. We find that adding more processors results in scaling performance and that there is negligible contention over resources. These results lead us to believe that the MLCA is a viable architecture for SoC solutions for multimedia applications.

The remainder of this paper is organized as follows. Section 2 gives an overview of the MLCA.

Section 3 describes the two applications and the compiler transformations applied to their code.

Section 4 presents our experimental evaluation. Section 5 describes related work. Finally, Section 6 gives some concluding remarks and directions for future work.

\section{THE MULTI-LEVEL COMPUTING ARCHITECTURE}

\subsection{The Architecture}

The MLCA is a 2-level hierarchical architecture that, at the lower level, consists of multiple processing units (PUs). A PU can be a full-fledged processor core (superscalar, VLIW, etc), a DSP, a block of FPGA, or some custom hardware. The upper level consists of a control processor (CP), a task dispatcher (TD), and a universal register file (URF). A dedicated interconnect links the PUs to the URF and to memory. A block diagram of the MLCA is shown in Fig. 1(a), which bears considerable similarity to an abstract microprocessor architecture as shown in Fig. 1(b).

The novelty of the MLCA stems from the fact that the upper level of the hierarchy supports out of order, speculative and superscalar execution of coarse-grain units of computation, which we refer to as tasks. It does so using the same techniques used in today's superscalar processors, such as register renaming and out of order execution, to exploit parallelism among instructions. This leverages existing superscalar technology to exploit task-level parallelism across PUs in addition to possible instruction-level parallelism within a PU.

The CP fetches and decodes task instructions, each of which specifies a task to execute. A task instruction also specifies

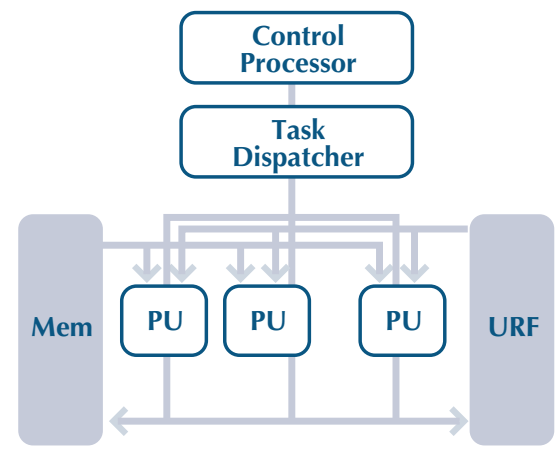

(a) Macro-architeture

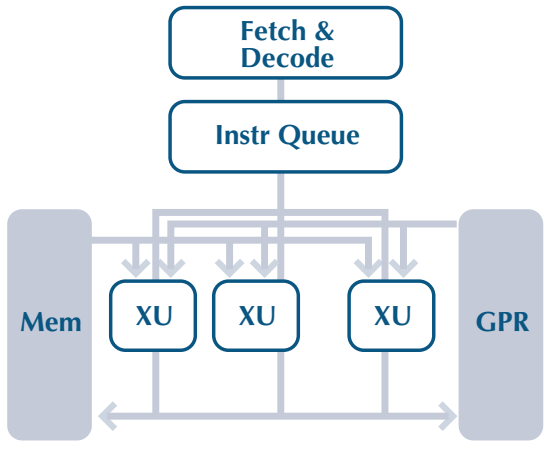

(b) Micro-architeture 
the inputs and outputs of the task as registers in the URF. Dependencies among task instructions are detected in the same way that dependencies among instructions are detected in a superscalar processor: by means of the source and sink registers in the URF. The $\mathrm{CP}$ renames URF registers as necessary to break false dependencies among task instructions. Decoded task instructions are then issued to the TD unit. Based on dynamic dependencies, tasks can be issued out of order, and may also complete and commit their outputs out of order.

Task instructions are enqueued in the TD unit in a similar way instructions are enqueued in the instruction queue of a superscalar PU. When the operands of a task instruction are ready, the task instruction is dispatched using a scheduling strategy to the PUs. The simplest such strategy dispatches instructions to PUs in a round-robin fashion. However, more dynamic strategies can also be used.

The MLCA is a template architecture. It does not specify the form of the interconnect among the PUs. Several implementations are possible, including buses, crossbars, and multi-stage interconnects (for certain configurations, we have worked on a dedicated multi-stage type interconnect called Octagon [11]). In addition, since inter-task dependencies are enforced by the $\mathrm{CP}$, and since data communication is primarily accomplished through the URF, there is no need to assume a particular memory architecture. The PUs may share a single memory, may each have their own private memory, or any combination of the two depending on the application.

\subsection{Programming Model}

The hardware features of the MLCA give rise to a natural programming model that is very similar to sequential programming. The MLCA programming model is layered. The bottom layer comprises the task bodies, or simply the tasks. Each task implements a given functionality and has defined inputs and outputs. A task can be a sequential C program, a block of assembly code executing on a programmable PU such as a processor or DSP core, or a predefined functionality of a non-programmable PU such as a hardware block.

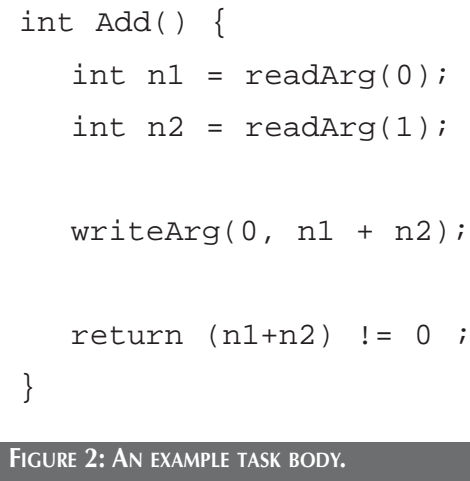

The top layer of the model is a single task-program that executes on the CP. It is a sequential program that specifies task instructions, and is expressed, for example, in a C-like language called Sarek. The language replaces function calls with task calls and adds explicit direction indications (in or out) for function arguments.

Figs. 2 and 3 illustrate the MLCA programming model. The task Add shown in Fig. 2 is expressed as a C function that computes the sum of two integers. The function has no formal arguments. Instead, it communicates with the Sarek program through an API, obtaining input data with a readArg call and writing results using an analogous writeArg call. For example, readArg(1) reads the second input to the function, while writeArg(0) writes the first output of the function. The task also returns a condition code that is written to a condition register in the $\mathrm{CP}$, and may be used in a Sarek program to make control decisions.

The main part of the corresponding Sarek program for the example is shown in Fig. 3. It makes four calls to the task Add. In each call, the variable names of the inputs and outputs of each task are specified. In addition, a direction indicator (in or out) is also specified for each variable. The second instance of the task Add must wait for the first instance to complete because of the true dependence caused by the use of the variable totwidth. However, the third and fourth instances of Add may proceed out of order with the first two even if they also write to and read from totwidth, and because there is no dependency with the conditional call to Div. The hardware automatically renames the 
(WAW) and Write-After-Read (WAR), the CP allocates new registers, allowing the tasks to run in parallel on separate PUs.

- Scheduling policy is independent from source code. Our layered approach fits in the layered model advocated by Thomas and Paul [14], where each layer (application, schedulers, resources) can be tuned independently to attain an optimal cost/performance ratio.

- Efficient communication. Tasks may exchange data through URF instead of relying on shared memory.

\section{THE APPLICATIONS}

We present two case studies of porting realistic multimedia applications to the MLCA. For each case, we describe the application, then the code transformations necessary to obtain good performance.

These code transformations build on well-known compiler analyses and optimizations, including data flow analysis [12], array privatization [18], code hoisting [12] and loop unrolling [12]. Hence, they can be easily incorporated into Sarek and C compilers.

\subsection{MAD}

MAD is an MPEG decoder that translates MPEG files into 16-bit PCM output [5]. We use a stripped-down version of the code, which does not include multithreading but retains the functionalities and code structure of the original application.

The input to MAD is a byte stream that represents a sequence of audio frames. Each frame consists of a frame header and frame data. The frame header contains configuration information such as audio layer type, channel mode, sampling frequency, stream bit rate, and the location of the frame's main data in the input stream. Since frames may be of different sizes, a frame header also contains the size of its corresponding frame.

The main data structure in MAD is a $\mathrm{C}$ structure called mad decoder. It contains global variables and three other $\mathrm{C}$ structures: mad_stream, mad_frame, and mad_synth. The mad stream structure stores the start and end addresses of the input stream in memory, a pointer to the start of the current frame being decoded, a pointer to the next frame to be decoded, and buffers used for decoding a frame. The mad_frame and mad_synth structures hold buffers for the decoded output and the PCM output for a frame, respectively. Thus, most of the pointers and buffers within mad_stream, as well as within the mad_frame and mad_synth structures, are re-used for the decoding of each frame.

Fig. 5 shows a break down of MAD's functionalities. The various data structures used by the program are first allocated and initialized. The file containing the input stream is then mapped to memory, and the frames are decoded one at a time until endof-file is reached. For each frame, the decoded output is copied to mad frame. The PCM output is synthesized and placed in the mad_synth structure. The structure is sent to either a file or the standard output. Finally, the input file is unmapped from memory, and the various structures are deallocated.

Frame decoding is performed in a number of steps. First, the header of the frame is read, and the length of the current frame is determined. Then, frame data is parsed from the input stream.

Data for successive frames do not synchronize with their respective headers and do overlap. Thus, the data header may point to data preceding it. These data are copied into a buffer in the the mad_stream structure, called main_data, before

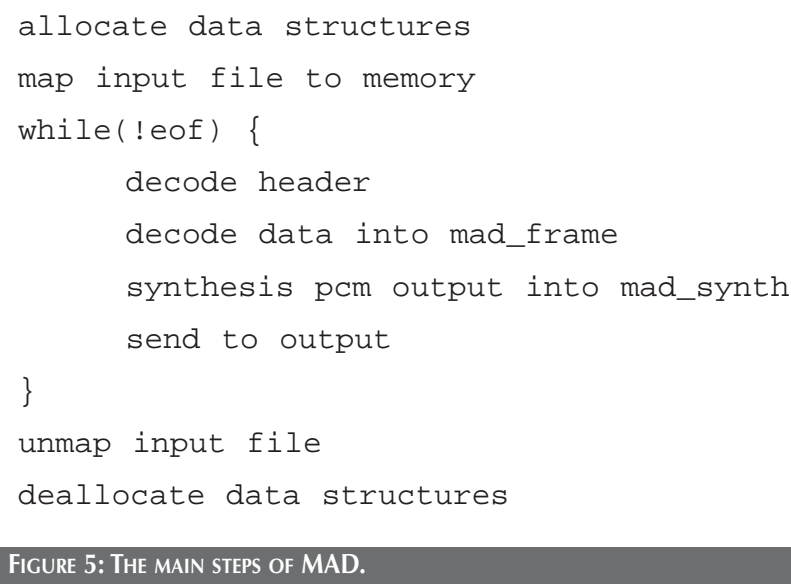

Figure 5: THE MAIN STEPS OF MAD. 
decoding. Copying and the decoding of the data are performed in three stages: load, decode, and preload. In load stage, the data in the current frame is copied from the input stream into main_data. In decode stage, the data in main_data are decoded. In preload stage, the data associated with the next header are moved from one part of main_data to another in preparation for processing of the next frame.

The first step in porting MAD to the MLCA is to determine the tasks and a corresponding Sarek program. This is a relatively simple step since the main function of MAD consists, for the most part, of calls to top-level functions, which become tasks. The initial Sarek program is depicted in Fig. 6.

The task Init corresponds to the function that creates and initializes all data structures contained in mad decoder. A pointer to this structure appears as a parameter to each function in the MAD program, and thus, as a parameter to each task in the Sarek program. Since each task reads and writes variables to/from this structure, the pointer is designated as both input and output to each task. The use of a pointer to mad_decoder as input and output arguments to each task results in two problems. The first is the false dependencies introduced by the use of a single pointer to reflect the dependencies among the tasks, in this case a pointer to mad_decoder. These dependencies are false because tasks use different parts of mad_decoder and thus can execute in parallel. The use of one pointer as both input and output causes the tasks to serialize, thus eliminating parallelism.

The second and more serious problem is introduced by the use of pointers as parameters to tasks. In order for a task to write to a memory location in a buffer or a structure, the pointer to this memory location must be an in argument to the task, even if the task is not reading the memory location. This creates an unnecessary true dependence, caused by the pointer itself. This also makes hardware renaming inapplicable, since every task using the pointer must get the original copy of the pointer value as a result of this "true" dependence. Furthermore, even if the pointer is removed as an in parameter, the hardware will rename the value of the pointer in the corresponding register, not the data pointed to by it, which is in memory.

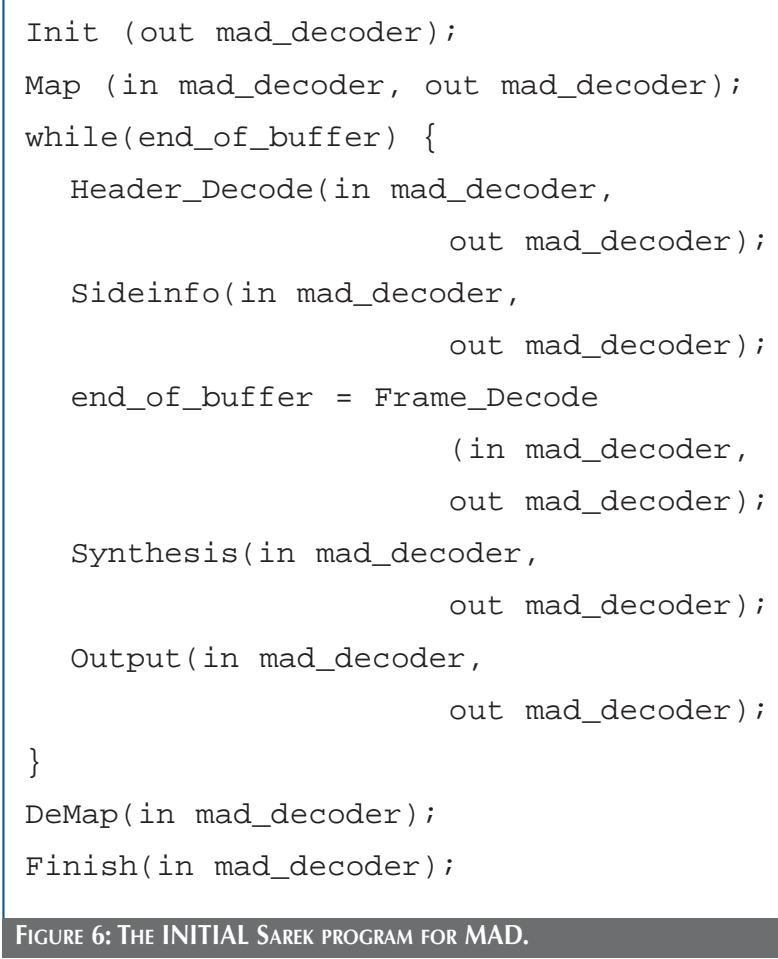

(a) Before de-aggregation

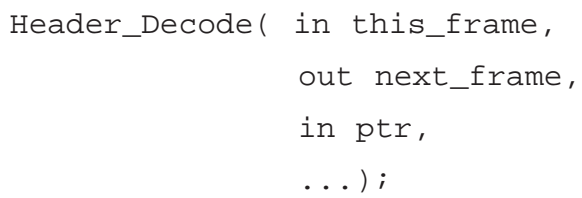

(b) After de-aggregation

\section{FIGURE 5: AN EXAMPLE OF PARAMETER DE-AGGREGATION.}

Consequently, we introduce a series of code transformations that are applied to both the Sarek program as well as tasks to overcome these problems. These transformations are described in the following sections.

\subsubsection{Task parameter de-aggregation}

The purpose of task parameter de-aggregation is to expose 
the elements of structures in the parameter list of tasks, thus eliminating false dependencies, and allowing the hardware to rename these parameters when appropriate. This is done by recursively replacing pointers to structures by components until all task parameters are of primitive types (e.g., int, float, int $*$, etc.). For each parameter, the direction of access (in and/ or out) must be determined. We compute, using standard data ow analysis techniques, upward exposed uses and downward exposed writes for each parameter in a task body. If there is an upward exposed read of a parameter, the parameter is designated as input; if there is a downward exposed write, the parameter is designated as output.

Fig. 7 shows the Header_Decode task header after transformation. It shows two (of many) elements of mad_ decoder now appearing as task parameters. The first two parameters, this_frame and next_frame, are integers. Thus, the hardware is able to eliminate false dependencies, from the use of this variable, among tasks. The third parameter, ptr, is a pointer to a buffer in memory, and as described above, causes a renaming problem. This problem must be resolved by renaming during compilation using buffer privatization, which is described next.

\subsubsection{Buffer privatization}

Buffer privatization is illustrated using the example in Fig. 8(a). Consider two tasks in the body of a while loop. The two tasks use the buffer buff. Output and anti dependencies prevent instances of the tasks in one iteration from executing in parallel with instances in another iterations. These dependencies result from the use of the same buffer area in memory by all iterations. It is possible to break the output and anti dependencies by privatizing the buffer, i.e., by giving each iteration a private copy of the buffer, as shown in Fig. 8(b). A new buffer is allocated at the beginning of each iteration using the new task Init and is deallocated at the of an iteration using the new task Finish. The hardware renames the parameter buff in each iteration of the loop, but now along with a corresponding private buffer. The task Finish is guaranteed not to complete until all tasks

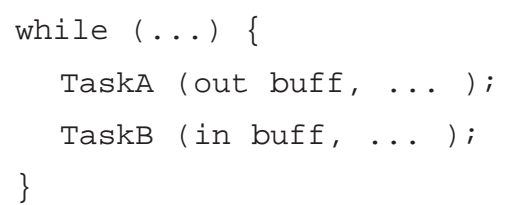

(b) After privatization

\section{FIGURE 8: AN EXAMPLE OF THE BUFFER PRIVATIZATION TRANSFORMATION.}

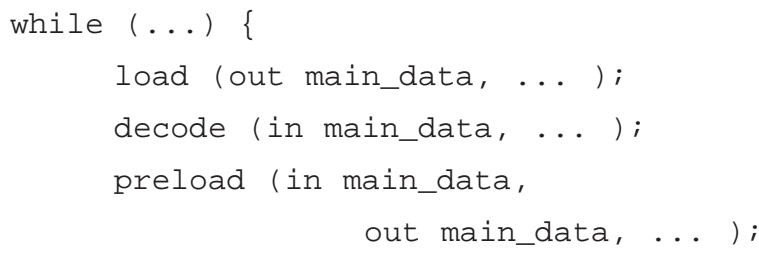

(a) Before replication

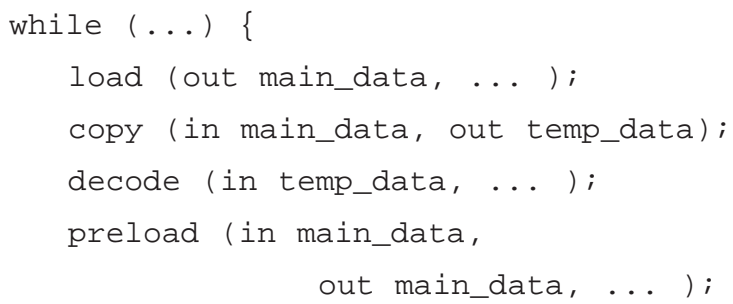

(b) After replication

in an iteration are complete through the addition of artificial dependencies.

In order for privatization of a buffer buff to be legal, it is 
necessary that every read to a section of buff be dominated by a write to the same section of buff in the same iteration. This transformation is similar to array privatization [18], which is successful in the context of automatic parallelization of loops [6]. The transformation requires buffer section analysis [18] as well as interprocedural alias analysis [15]. This transformation is particularly effective in MAD because buffers within all the main structures are re-used for the decoding of each frame.

\subsubsection{Buffer replication}

In some cases, not all reads to sections of a buffer are dominated by writes to the same sections in an iteration, making buffer privatization inapplicable. Nonetheless, parallelism can be introduced by overlapping the execution of tasks in one iteration with the execution of tasks in subsequent iterations. The transformation used to accomplish this is called buffer replication.

Buffer replication is illustrated using the example shown in Fig. 9(a). Consider the three tasks, Load, Decode, and Preload, all of which use the buffer main_data. They are part of the
Frame_Decode component of MAD. Load writes only to the second half of buff; Decode reads the entire buffer; Preload reads the second part of buff and writes to its first half. Privatization is not possible because, in an iteration, Decode reads buffer data written in the previous iteration by Preload. The dependencies among the tasks cause their execution to serialize as shown in Fig. 10(a).

In order to achieve some parallelism, the main_data buffer is replicated and copied into the buffer temp_data in copy, as shown in Fig. 9(b). This copy is given to Decode, breaking the anti-dependence between Decode and Preload, thus allowing them to execute in parallel. It should be noted that a new copy of temp_data is allocated every iteration of the loop and that the hardware automatically renames temp_data every iteration. The execution of the tasks after buffer replication is shown in Fig. 10(b). There is now overlap among Decode task instances in different iterations because each instance uses a different buffer.

Buffer replication requires the same set of analysis required for buffer privatization.

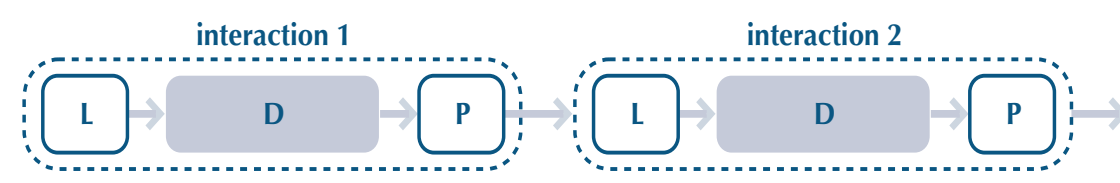

(a) Execution of tasks is serialized by dependences.

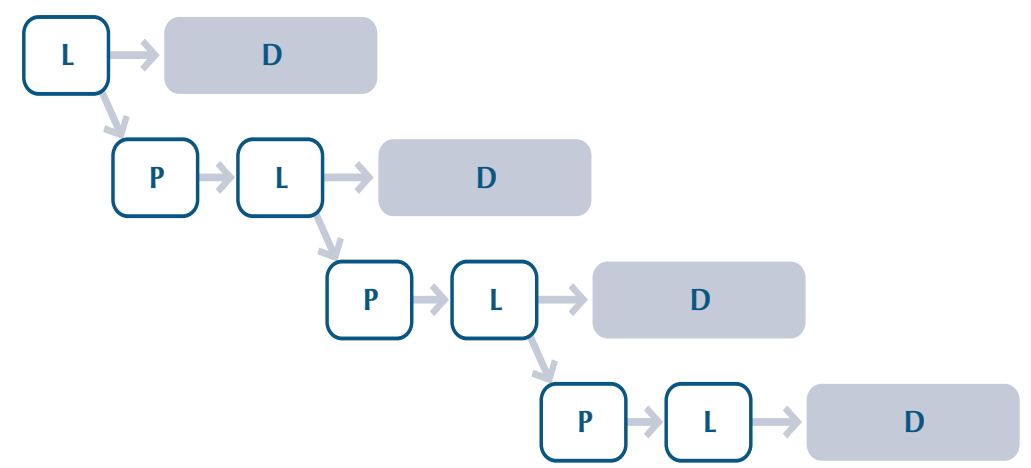

(b) Parallel execution is achieved by buffer replication. 


\subsubsection{Task splitting}

This transformation aims to increase the parallelism among tasks by splitting a task into multiple tasks. For example, the Frame_Decode task consists of calls to three functions: Load, Decode, and Preload, that perform the actions described in the previous section. However, these functions are not tasks. Thus, the Frame_Decode task is split by transforming these three functions functions into separate tasks that are now part of the Sarek program.

\subsubsection{Code hoisting}

A task cannot start until all its input parameters are available. Thus, it is desirable to write the output parameters of a task as early as possible to allow waiting tasks to proceed. We apply code hoisting [12] to the body of a task to move calls to writeArg to the earliest point possible.

\subsubsection{Loop unrolling}

The MLCA can support out of order speculative execution Nonetheless, we apply loop unrolling to the main loop in the MAD Sarek program in order to increase the amount of task parallelism. The body of the main loop is copied as many times as the number of processors used $(P)$, and the loop condition is checked every $P$ iterations.

\subsection{FMR}

FMR is an audio application that performs FM demodulation on a 16-bit input data stream, producing a 32-bit output data stream. The input stream consists of data packets of 1536 bytes each. The main steps of the program are performed by some 70 calls to 16 functions in a loop in the main functions, making the derivation of a Sarek program straightforward. The same set of transformations used for MAD are alos used for FMR to realize parallelism among tasks on one loop iteration, as well as among instances of tasks in successive iterations.

\section{EVALUATION AND ANALYSIS}

We developed a timed functional model of an instance of MLCA in about 6,000 lines of $\mathrm{C}++$ /SystemC.
The model reflects the overall structure of MLCA, with a Control Processor, Task Dispatcher, Universal Register File and some PUs, with associated PU caches and shared or distributed memory.

In this instance of MLCA, we are using ARM processors as the Processing Units.

The model instantiates the desired configuration at runtime. Parameters include number and type of PUs, URF size, number of renaming registers, cache and memory configuration and associated latencies, and relative speed of CP, TD, and PUs.

Each PU can be configured with a cache and a combination of local and global memory. The interconnect adds a constant delay, and the memory model implements a simple contention mechanism, where the requests are enqueued in order and dequeued at a given rate. The model of URF contention is similar. The caches are write-through; thus memory always contains the most up-to-date copy of data. Cache lines need not be invalidated on every write to maintain consistency; rather, caches can be maintained consistent by invalidating the entire cache at the end of task execution on the corresponding processor. We make use of 8-way set-associative caches with cache block size of 8 bytes and only global memory. We assume 4 ports to the URF (and thus renaming registers), and similarly 4 ports to the global memory.

We used the simulator to evaluate the performance of MAD and FMR. MAD is used to decode an input MP3 song file, which consists of 126 frames with 2 channels, $22050 \mathrm{~Hz}$ sample rate, and a $40 \mathrm{Kbps}$ bit rate. It executes in 137312446 cycles on one processor. FMR is used to decode 21 input packets, each consisting of 1536 bytes. It executes in 112468135 cycles on one processor.

Fig. 11 shows the speedup of the two applications as a function of number of processors. The speedup of each application at $P$ processors is defined as the ratio of the execution time of the application on one processor to the execution time of the application at $P$ processors. The speedup is shown for various numbers of renaming registers (see below). The figure shows that each application exhibits scaling speedup, which is 


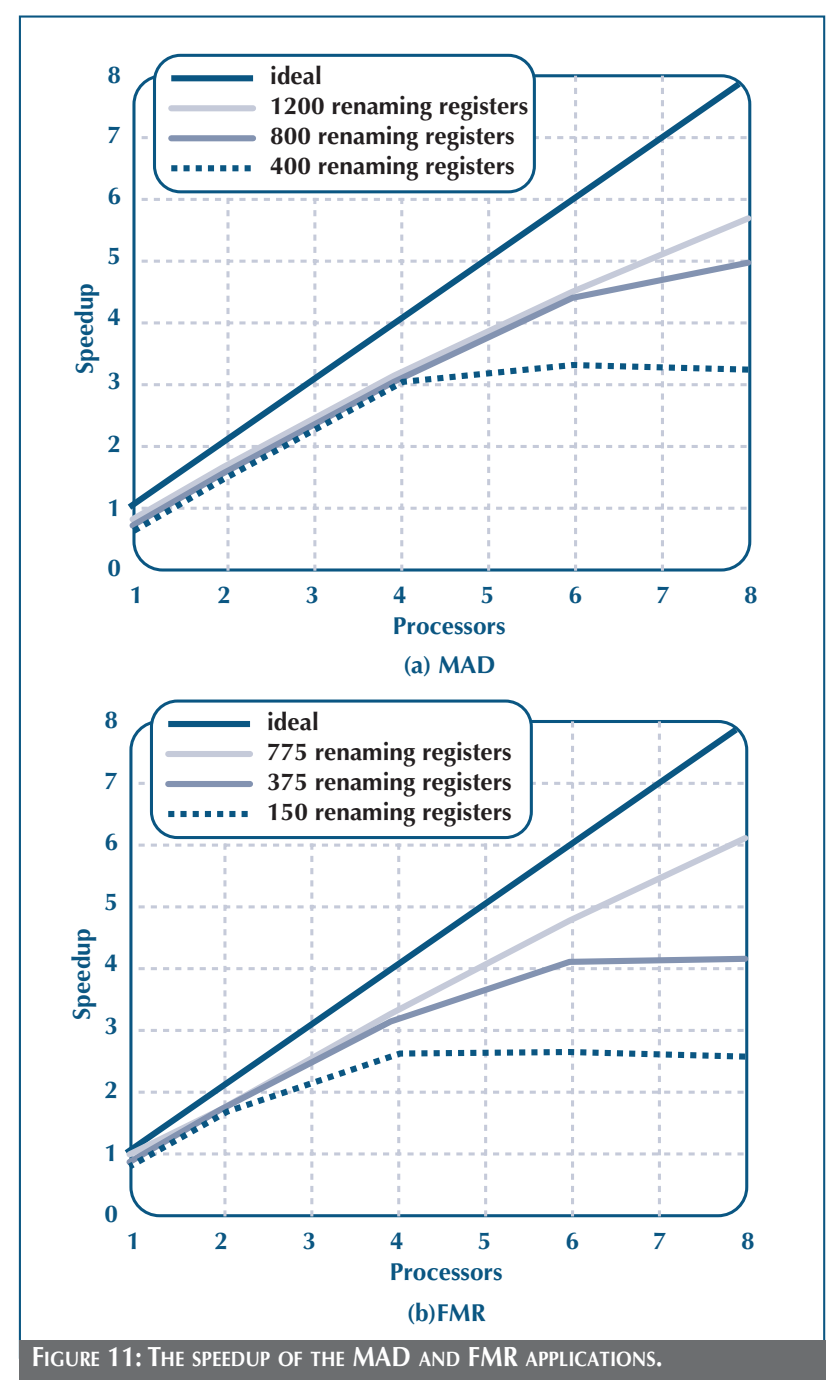

relatively close to ideal when the number of renaming registers is suciently large. The figure also shows that the speedup of MAD at 1 processor is slightly less than 1 . This reflects the overhead that is incurred through the code transformations. For example, the transformed code of each application allocates and deallocates buffers during its execution, which does not occur in the sequential un-transformed application. Nonetheless, as the number of processors is increased, the benefits of parallelism outweigh this overhead.

Fig. 11 also shows that speedup of each application depends on the number of renaming register; in general, the more renaming registers are available, the higher the speedup. Fig. 12 further explores this issue. It shows the speedup of MAD and FMR as a function of the number of renaming registers for different numbers of processors. The figure shows that the speedup of each application experiences a noticeable increase up to a certain lbreakpoint", then remains relatively at after-wards. This breakpoint is different for each application and also for each number of processors.

This behavior of application speedups can be explained as follows. The increase in the number of renaming registers allows more tasks to execute in parallel1. However, the increase in the number of registers will have an impact only if it enables the execution of more tasks. When all false dependencies are removed by the addition of enough renaming registers, the speed of execution of each application is dictated by the true dependencies among its tasks. The addition of more renaming registers does not lead to the execution of more tasks. Thus, the speedup improves up to the point where all false dependencies are broken and then remains at. Similar to renaming registers, the availability of processors dictates the maximum number of tasks that can execute, and the break-point is also a function of the number of processors. Since the number of false dependencies is different for each application, the breakpoint is also different for each application.

However, it can also be noticed in Fig. 12, especially for 8 processors, that the addition of more renaming registers sometimes decreases the speedup rather than improves it. This is due to the impact of additional registers on the scheduling of tasks, as explained in details in [10].

It is important to note that the potentially large number of renaming registers poses no performance bottleneck in the toplevel of the MLCA. The granularity of tasks executing on the PUs is several orders of magnitude bigger than the URF register access time. Thus, URF registers are not likely to be accessed every cycle, and they need not be as fast as registers within the PUs. Indeed, as one indicator, we experimented with the impact of the number of access ports to the URF. In both applications, 


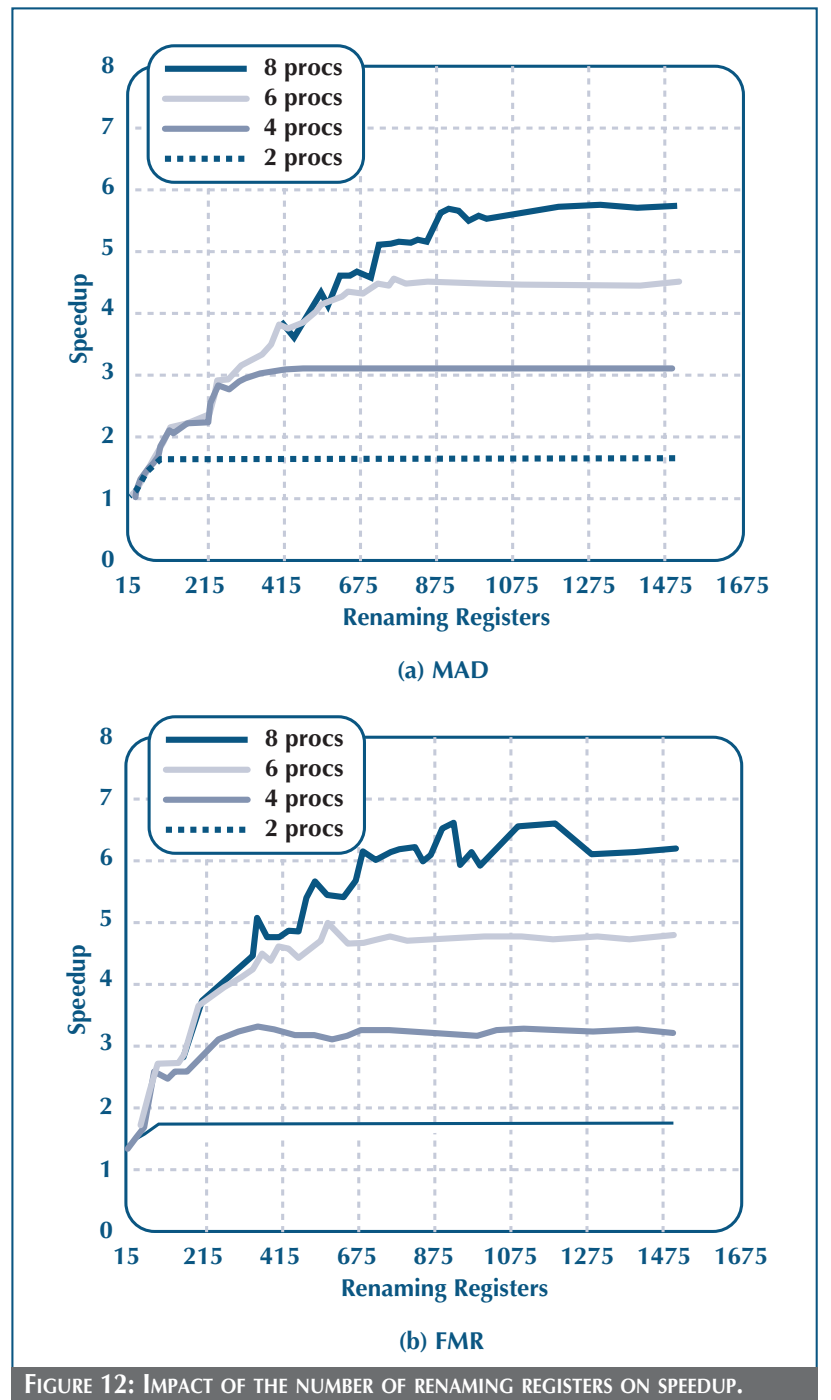

changing the number of access ports from 1 to 8 had negligible impact on performance. This is also the case for the number of memory ports. Changing the number of memory ports has negligible impact on the speedup of the two applications, indicating that both the URF and the memory are not strongly contended for.

\section{RELATED WORK}

MLCA's tasks bear some similarities with the user-defined functions of GLU [8], a coarse-grained dataflow language. The combination of tasks in a sequential language (C) and the toplevel dataflow exhibited very good performances.

The MLCA uses superscalar technology, which was pioneered by Tomasulo [17] in 1967. Hennessy and Patterson [7] and Smith and Sohi[16] offer excellent overviews of modern superscalar architectures.

The extension of superscalar principles to tasks instead of instructions and the associated scheduling was explored by Verians, Legat et al. [19, 21]. The core of their work is an efficient implementation of the instruction queue, which has to check on the operands availability to perform the out of order dispatching. However, they rely on a shared memory instead of a shared register file for inter-task communication.

There exists a number of SoC systems that use multiple processing units for multimedia and other applications. However, they differ from the MLCA in their programming model.

Daytona's scalable DSP architecture [13] features a splittransaction bus for communication and cached semaphores for synchronization. The programming model [9] is also layered, separating tasks and top-level control flow. The dynamic scheduler is implemented in a run-time kernel and is configurable.

The picoChip [4] is a cascadable reconfigurable architecture of array processors intended for $3 \mathrm{G}$ wireless communications. However, in contrast to the MLCA, which is programmed in a quasi-sequential model, the picoChip is programmed using VHDL.

Cradle's Technologies's $3 S O C$ is a shared-address space multiprocessor SoC. It consists of a number of processor clusters that are connected by two levels of buses [1]. The system provides 32 semaphore registers for synchronization, which must be explicitly used in a parallel program. In contrast, our MLCA automates the synchronization of tasks through the URF, providing a programming model that is closer to sequential

\footnotetext{
1 - The renaming registers allow the hardware to break false inter-task dependencies, and thus to issue more task instructions in parallel.

The hardware stops issuing instructions when it runs out of renaming registers.
} 
programming. The code transformations used to improve the performance of programs for the MLCA build on a number of well-known compiler analyses and optimizations, including data ow analysis [12], array privatization [18], code hoisting [12], and loop unrolling [12].

\section{CONCLUSIONS}

The MLCA uses existing superscalar techniques to exploit parallelism among tasks of coarse-grained computations automatically. It allows tasks to be executed out of order; the hardware synchronizes tasks based on their true dependencies and eliminates false dependencies using register renaming.

This hardware support gives rise to a programming model that is very close to sequential programming: individual tasks are expressed in a sequential language like $\mathrm{C}$, while a task program written in a $\mathrm{C}$-like language initiates the execution of these tasks.

We evaluated an instance of the MLCA using simulation and two realistic multimedia applications. The modularity of the applications makes it relatively straightforward to write an initial task-level program. Code transformations are used to improve performance. Our results show that both applications exhibit good performance. Furthermore, the performance appears to be scalable, demonstrating the architecture's potential.

Our future work will address a number of issues, including: design and evaluation of a memory hierarchy for the MLCA, task definition and formation, task scheduling, and system evaluation using industry-standard applications.

\section{REFERENCES}

[1] 3SOC documentation -

3SOC 2003 hardware architecture, cradle technologies, Inc., March 2002.

[2] 3SOC programmer's guide, cradle technologies, Inc., March 2002. http://www . cradle. com.
[3] http://www . gnu.org.

[4] http://www.picochip.com.

[5] http://www . underbit.com/products $/ \mathrm{mad} /$.

[6] W. Blume et al. "Parallel programming with Polaris". "IEEE Computer", 29(12):78-82, 1996.

[7] J. Hennessy and D. Patterson.

Computer Architecture: A Quantitative Approach. Morgan Kaufmann, 2003.

[8] R. Jagannathan. "CoARse-Grain Data ow Programming of Conventional Parallel Computers". "Advanced Topics in Data flow Computing and Multithreading".

IEEE Computer Society Press, pp. 113-129, 1995.

[9] A. Kalavade, J. Othmer, B. Ackland, and K. J. Singh. "SOFTWARE ENVIRONMENT FOR A MULTIPROCESSOR DSP". In Proc. of the Design Automation Conference, pp. 827-830, 1999.

[10] F. Karim, A. Mellan, U. Aydonat, T.S. Abdelrahman, B. Stramm, and A. Nguyen. The Hyperprocessor: a template system-on-chip architecture for embedded multimedia applications.

Workshop on Application Specific Processors, pp. 66-73, 2003.

[11] F. Karim, A. Nguyen, S. Dey, and R. Rao. “ON-CHIP COMMUNICATION ARCHITECTURE FOR OC-768 NETWORK PROCESSORS". In Proc. of the 38th Design Automation Conference, pp. 678-683, 2001.

[12] S. Muchnick. "Advanced Compiler Design AND IMPLEMENTATION”. Morgan Kaufmann, 1997. 
[13] C. Nicol et al. "A SingLe-CHIP, 1.6-BILLION, 16-B MAC/s MULTIPROCESSOR DSP”. IEEE Journal of Solid-State Circuits, 35(2), March 2000.

[14] J. Paul and D. Thomas. "A LAYERED, CODESign VIRTUAL MACHINE APPROACH TO MODELING COMPUTER SYSTEMS”. In Proc. of Design Automation and Test in Europe, pp. 522-528, 2002.

[15] R. Rugina and M. Rinard. "Automatic PaRALLELIZATION OF DIVIDE AND CONQUER ALGORITHMS". In Proc. of Principles and Practice of Parallel Programming, pp. 72-83, 1999.

[16] J. Smith and G. Sohi. "The Microarchitecture OF SUPERSCALAR PROCESSORS". Proc. of the IEEE, 83:1609-1624, 1995

[17] R. Tomasulo. “AN EFFICIENT ALGORITHM FOR EXPLOITING MULTIPLE ARITHMETIC UNITS".

IBM Journal of R \& D, 11:25-33, 1967.

[18] P. Tu. "Automatic Array Privatization and Demand Driven Symbolic Analysis". $\mathrm{PhD}$ thesis, Department of Computer Science, University of Illinois at Urbana-Champaign, 1995.

[19] X. Verians, J.-D. Legat, J.-J. Quisquater, and B. Macq. "A NeW PARALLELISM MANAGEMENT SCHEME FOR MULTIPROCESSOR SYSTEMS". Lecture Notes in Computer Science, 1557:246-256, 1999.

[20] D. Wall. "Limits of InSTRUCTION-LEVEl PARALLELISM". In Proc. of Architectural Support for Programming Languages and Operating Systems, pp. 176-189, 1991.
[21] J.-J. Q. X. Verians, J-D. Legat. "EXTENSION DU PRINCIPE SUPERSCALAIRE AU TRAITEMENT DE BLOCS D'INSTRUCTIONS".

In Congrès 2001 de l'Association Francaise des Sciences et Techniques de l’Information, pp. 113\{125, 2001. 